\title{
Advantages and Controversies in the Era of Intrarenal Volumetry
}

\author{
Michael Pedersen ${ }^{\mathrm{a}, \mathrm{b}} \quad$ Kristian Karstoft $^{\mathrm{a}-\mathrm{c}}$ Anders Lødrup $^{\mathrm{a}-\mathrm{c}}$ Bente Jespersen $^{\mathrm{b}, \mathrm{d}}$ \\ Jens R. Nyengaard ${ }^{\text {b, }}$, \\ ${ }^{a}$ MR Research Center and ${ }^{b}$ Institute of Clinical Medicine, ' Stereology and Electron Microscopy Laboratory and \\ Center for Stochastic Geometry and Advanced Bioimaging, and d Department of Nephrology, Aarhus University \\ Hospital, Skejby, Denmark
}

\section{Key Words}

Animal models $\cdot$ Contrast-enhanced MRI $\cdot$ Kidney biopsy •

Glomeruli $\cdot$ Microscopy $\cdot$ MRI volumetry $\cdot$ Pathology

\begin{abstract}
Background/Aims: Ultrasound is the preferred imaging modality in nephrology. In many kidney diseases, however, more accurate methods are needed to distinguish between relevant intrarenal structures. MRI could be a solution, although the use of MRI contrast has caused severe complications in some cases, and invasive kidney biopsies may follow, even though such small specimens traditionally provide inaccurate quantitative data. We evaluated the usefulness of MRI volumetry and quantitative kidney biopsies to assess glomerular number and volume as well as cortical volume. Methods: We specifically highlight an experimental study in which different MRI scans were performed in healthy pigs as well as pigs with unilateral ureteral obstruction to assess intrarenal volume. Single-kidney glomerular filtration rate (GFR) was measured using ureteral cannulation and ${ }^{51} \mathrm{Cr}$ EDTA. Kidney biopsies were taken and evaluated employing stereological techniques to measure number and volume of glomeruli. Pigs were sacrificed and kidneys were removed for stereological analysis. Non-contrast-based MRI intrarenal
\end{abstract}

volumes were - without significant difference to intrarenal volumes - obtained using contrast-enhanced MRI and ex vivo techniques. Results: Kidney biopsies gave valid estimates regarding quantitative parameters, such as mean number and volume of glomeruli in the cortex. Different structural parameters correlated with kidney GFR with high, although varying, correlation coefficients. Conclusion: Noncontrast MRI is suitable for estimating intrarenal volumes in healthy and diseased kidneys. We advocate further research in diagnostic modalities combining MRI and biopsies. Major challenges are the cortical architecture and heterogeneous distribution of glomeruli within the kidney.

Copyright $\odot 2011$ S. Karger AG, Basel

\section{Introduction}

This article advocates the use of complementary MRI and stereology methods in nephrologic diagnostics. Today, ultrasound is the preferred imaging modality, and whole-kidney volume is based on a pole-to-pole length [1-3]. Kidney volume is a highly relevant parameter as many diseases and particularly chronicity are associated with changes in kidney size, whereas pole-to pole length only gives a crude estimate of renal volume [4]. Further-

\section{KARGER}

Fax +4161306 1234

E-Mail karger@karger.ch

www.karger.com
(C) 2011 S. Karger AG, Basel

0250-8095/11/0337-0040\$38.00/0

Accessible online at:

www.karger.com/ajn
Jens R. Nyengaard, MD, Dr. Med. Sc.

Stereology and Electron Microscopy Laboratory

Building 10G, Aarhus University Hospital NBG

Noerrebrogade 44, DK-8000 Aarhus C (Denmark)

Tel. +45 8949 9928, E-Mail nyengaard@ki.au.dk 
more, the normal range is large and influenced by factors such as gender, age, and body shape, which should be taken into account to arrive at the most significant conclusions and information [3]. More importantly, traditional ultrasound diagnostic measurements provide little information about changes in intrarenal segments. Conversely, light, electron and immunofluorescence microscopy (of biopsy samples) have been invaluable in renal pathology for the study of glomerular structure, ultrastructural details of the glomerular capillary wall and the glomerular basement membrane, tubuli and the interstitium [5-7]. However, traditional biopsies only contain 10-20 glomeruli out of approximately 1 million, and quantitative assessments are limited, although they are important in the assessment of most kidney diseases, particularly in glomerulonephritis $[8,9]$, allograft nephropathy [10], and the evaluation of chronic injury in parenchymal renal diseases [11]. When the kidneys shrink due to fibrosis and tissue destruction, a biopsy is associated with a high risk of bleeding and frequently provides limited information. Renal artery stenosis reduces flow and thereby triggers autoregulatory mechanisms, particularly renin secretion, in the affected kidney, leading to increased blood pressure in the systemic circulation, which also affects the contralateral kidney [12]. Over time, loss of renal parenchyma in the stenosed kidney may occur, and microangiopathy may develop in the glomeruli of the non-stenosed contralateral kidney [13, 14]. This pathological process results in reduced volume in the kidney with the stenosed artery [15], whereas the contralateral, non-stenosed kidney may grow as a physiological compensation, yet with time, shrinkage may also occur as a result of hypertensive nephropathy. Very early in the course of diabetic nephropathy, kidneys may become enlarged [16]. In all kidney diseases, cortical volume $\left(\mathrm{V}_{c}\right)$ might offer more information than kidney length. In polycystic kidney disease, enlargement of the kidney volume due to cyst formation indicates progression of the disease, possibly leading to renal failure, and whole-kidney volume assessment has been shown to be a predictor and a method to monitor progression [17]. In other renal diseases, kidneys shrink with disease progression and loss of glomerular filtration rate (GFR) [18]. Renal volume is expected to be a much more sensitive marker for progression and GFR loss than the traditionally measured kidney length, but $\mathrm{V}_{\mathrm{c}}$ measurements may be even better.

The effects of ureteral obstruction (UO) on intrarenal volume are inherently complex and depend on many factors. An acute obstruction will most likely lead to increased total renal volume due to dilation of the pelvic region, whereas a more chronic obstruction potentially will lead to decreased total renal volume due to cortical shrinkage, which may depend on the magnitude of the obstruction [19]. Therefore, in UO kidneys, intrarenal volumetric assessment would be of particularly high diagnostic value. Renal allograft nephropathy could be attributed to vascular complications, acute or chronic parenchymal disease caused by rejection, side effects of immunosuppression, recurrence of primary disease or hypertension, and urinary obstruction may prevail as well. In addition, intrarenal volumetry may help in the differential diagnosis of diseases in both native kidneys and renal allografts.

Hence, intrarenal segmental volumes rather than whole-kidney measures will provide superior information. In fact, whole-kidney volumetry may not at all imply unequivocal renopathological conclusions. For example, injury of the glomerular microvasculature mediated by non-immunologic processes, which are often the underlying mechanisms of progressive deterioration of renal function in patients with a variety of renal disorders, will initiate functional and structural adaptations with secondary loss of functioning nephrons [20]. Experimental studies revealed that intrarenal vasodilatation leads to a significant compensatory hyperfiltration via increased glomerular capillary pressure and plasma flow, a functional state that is accompanied by a parallel increase in glomerular volume attained chiefly by expansion of matrix components and an increase in the number of endothelial and mesangial cells [21].

Concomitantly, in most renal diseases, quantitative information about total number of glomeruli $\left(\mathrm{N}_{\text {glom }}\right)$ and total volume of glomeruli $\left(\mathrm{V}_{\text {glom }}\right)$ seems to be of significant importance. Interestingly, human autopsy studies evaluated by stereology have shown a negative correlation between $\mathrm{N}_{\text {glom }}$ and severe diabetic glomerulopathy [22, $23]$ together with an increased $V_{\text {glom }}$ in patients with insulin-dependent diabetes mellitus with albuminuria [24]. We used a similar approach in a UO model in pigs to show that both $\mathrm{N}_{\text {glom }}$ and $\mathrm{V}_{\text {glom }}$ were lowered in obstructed kidneys compared to a control group. This quantitative technique also revealed a decreased cortical glomerular volume fraction in stable human kidney grafts [25].

In this paper, we systematically communicate our experimental findings with combined intrarenal volumetry by MRI and stereological evaluations of renal biopsies, motivating this approach for clinical purposes. We will demonstrate how in vivo volume measures can be derived in a (semi-)automatic and user-independent way with high accuracy, and whenever both MRI and biopsy sam- 


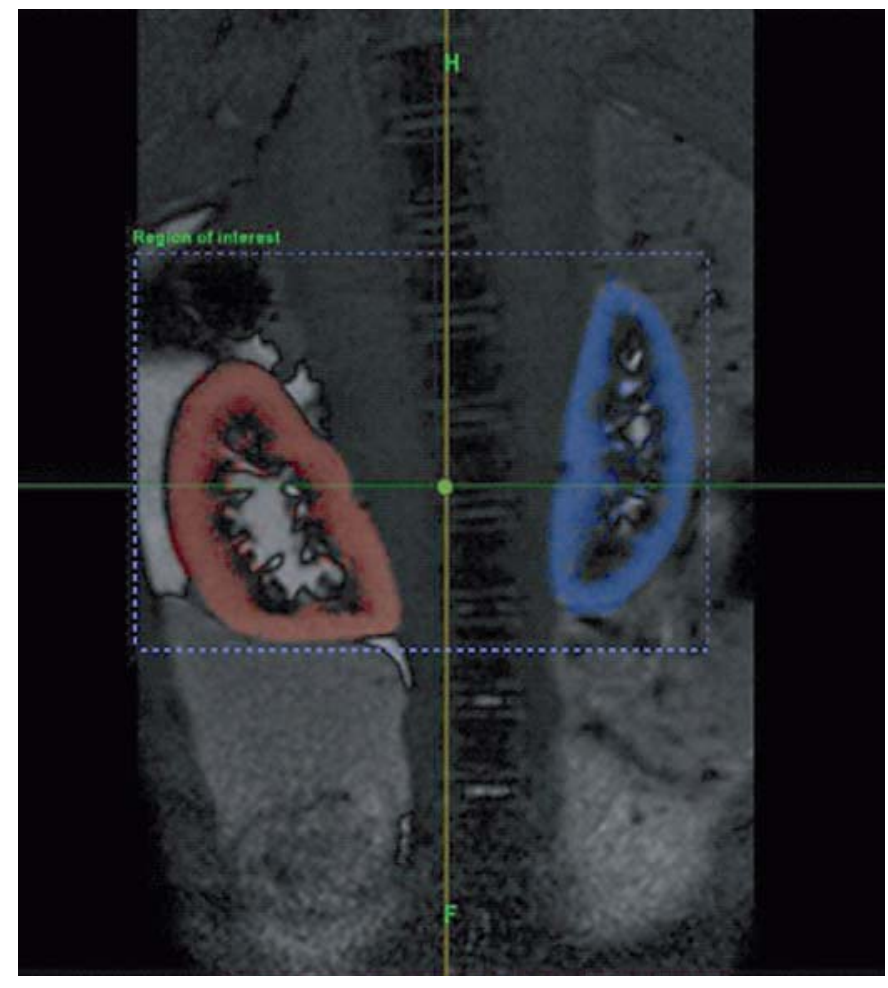

Fig. 1. Segmentation of the left and right renal cortex using a noncontrast agent MRI method. Black represents the medulla, and the inner bright area represents the renal pelvis.

ples are available, we will demonstrate how these seemingly different methods can provide complementary information about different structural parameters as well as renal function.

\section{Renal MRI Combined with Stereological Methods}

Absolute volume can be directly determined using several imaging techniques (e.g. MRI or CT), where the whole kidney, renal vessels, and renopathological structures can be depicted in three dimensions and entirely immersed in a spatial grid by virtual imaging techniques. Note, however, that the precise incidence of contrast-induced nephrotoxicity following intravenous contrast administration remains unclear in vivo both by MRI (due to the advent of the gadolinium-induced nephrogenic systemic fibrosis) and CT (iodinated contrast media). Thus, we propose a completely non-invasive MRI procedure, with a fast, efficient and reliable protocol, as the imaging method of choice to extract intrarenal volumes. In brief, differences in the intrinsic magnetic properties of water between renal segments can be used to selectively visualize intrarenal segments. MRI is capable of extracting these details on a three-dimensional (3D) grid by exploiting the magnetic relaxation properties of water in the renal cortex, medulla and pelvis using a strong $\mathrm{T}_{1^{-}}$ weighted sequence. Most available clinical MRI systems comprise acquisition techniques governed by inversion recovery spin echo sequences that are particularly sensitive to differences in $\mathrm{T}_{1}$ values. No contrast media are needed. Further, we introduce stereological methods based on sound mathematical and statistical principles to obtain quantitative data for the estimation of $\mathrm{N}_{\text {glom }}$ and $\mathrm{V}_{\text {glom }}[23,26]$. Stereology makes use of 'the physical fractionator', where the number of objects (e.g. $\mathrm{N}_{\text {glom }}$ in the whole renal cortex) is obtained from estimates of fractions sampled from that whole structure (e.g. a known fraction of the cortex); 'the disector' is a technique for unbiased sampling of objects using section planes (e.g. 2 consecutive slices of the renal cortex), and 'the Cavalieri principle' can be used for estimating the volume of structures divided into parallel planes (e.g. a kidney or a glomerulus divided into several parts, of which each profile area can be estimated).

\section{Methods}

\section{UO Model}

To demonstrate the usefulness of the proposed MRI and stereology means, we draw attention to our motivating findings reported in a UO animal model [27-29]. As mentioned, UO is followed by several renal complications and is known to upregulate the renin-angiotensin system, leading to intrarenal hypoxia and resulting in loss of nephrons over time [30]. In a reversible unilateral UO pig model, with ureteral ligature being induced by occlusion, we have previously observed marked changes in the cortical and medullary oxygenation in parallel with a significant reduction in the number of nephrons $[31,32]$.

\section{MRI: Cortical Volume}

Multi-slice MRI was performed in this UO pig model using a respiratory-gated 2D IRTSE MRI sequence (field-of-view $=320 \times$ $320 \mathrm{~mm}^{2}$, matrix $=256 \times 256$, slice thickness $=3 \mathrm{~mm}$, time of recovery $=4,500 \mathrm{~ms}$, echo time $=8 \mathrm{~ms}$, time of inversion $=100$ $900 \mathrm{~ms}$; 1.5-tesla MRI system). Volumetric data of a single MRI slice are demonstrated in figure 1, with a clear contrast between renal cortex and surrounding segments, allowing a straightforward semiautomatic segmentation of the renal cortex. In the same way, it is possible to define areas of the medulla and pelvis using dedicated volumetric segmentation software. Based on this technique, intrarenal areas were semiautomatically segmented using a $3 \mathrm{D}$ watershed-based algorithm. In this application, $\mathrm{V}_{\mathrm{c}}$ could be calculated from the sum-of-areas principle [33]. Interestingly, we found that $V_{c}$ in both healthy and UO kidneys could be measured 
Fig. 2. a Digital image of a biopsy sample. b The area of the sampled glomerulus profile can be estimated using the known lengths of the test lines in the $2 \mathrm{D}$ nucleator.
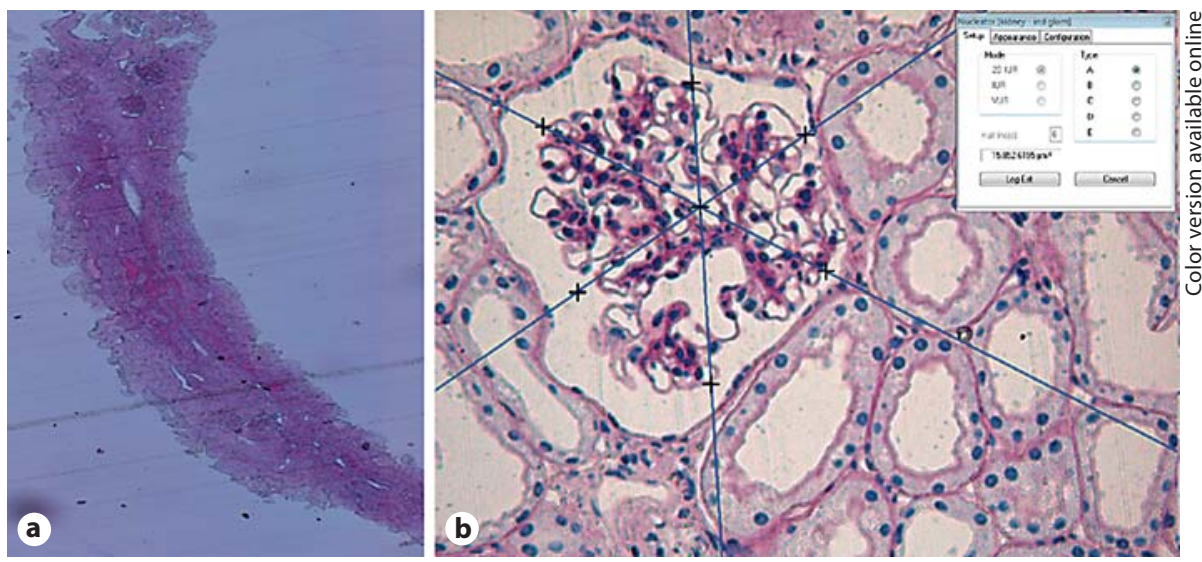

accurately with MRI using semiautomatic as well as manual segmentation, and measurements were obtained with a high intraand inter-observer reproducibility (covariance level <5\%) [29].

\section{Stereology: Glomerular Number and Volume}

In the same UO porcine model, 6 biopsies were sampled from each kidney, and estimates of $\mathrm{N}_{\text {glom }}$ and $\mathrm{V}_{\text {glom }}$ of both healthy and obstructed kidneys were derived by stereology. In short, each biopsy was divided into a medullary part and a cortical part using a stereomicroscope. The mass of the cortical part was measured, and a volume for each cortical biopsy was calculated assuming that the mass density of renal cortex was $1.04 \mathrm{~g} / \mathrm{cm}^{3}$. The biopsies were embedded in plastic (Technovit 7100) and cut exhaustively into 20- $\mu \mathrm{m}$-thick slices. Digital images were obtained from all slices using analysis software (newCAST, Visiopharm, Denmark), and glomeruli were sampled using 'the disector principle' (fig. 2). The volume of a single glomerulus was estimated on the basis of the glomerular profile area and 'the Cavalieri principle'. $\mathrm{N}_{\text {glom }}$ was then given by the equation: $\mathrm{N}_{\text {glom }}=\mathrm{Nv}_{\text {glom } / \text { cor }} \times \mathrm{V}_{\text {cor }}$, where $\mathrm{Nv}_{\text {glom/cor }}$ is the cortical glomerular density obtained from the $\mathrm{N}_{\text {glom }}$ in the biopsy and from the biopsy volume, and $\mathrm{V}_{\text {cor }}$ is the renal cortical volume. The total $V_{\text {glom }}$ was then given by the equation: $\mathrm{V}_{\text {glom }}=\mathrm{Vn}_{\text {glom }} \times \mathrm{N}_{\text {glom }}$, where $\mathrm{Vn}_{\text {glom }}$ is the mean glomerular volume given by $\mathrm{N}_{\text {glom }}$ and $\mathrm{V}_{\text {glom }}$ within the biopsies. Ex vivo kidneys, processed for microscopy, underwent stereological analysis using the physical fractionator, the disector and test point systems for control methods regarding $\mathrm{N}_{\text {glom }}$ and $\mathrm{V}_{\text {glom }}$. In this study, ex vivo estimates regarding $\mathrm{V}_{\text {cor }}$ and $\mathrm{N}_{\text {glom }}$ were used to calculate $\mathrm{N}_{\text {glom }}$ and $\mathrm{V}_{\text {glom }}$ from biopsy, respectively. Determination of both $\mathrm{V}_{\mathrm{c}}$ and $\mathrm{NV}_{\text {glom/cor }}$ makes it possible to calculate total $\mathrm{V}_{\mathrm{glom}}$.

\section{Results}

\section{Volumetric Measures Are Associated with Intrarenal} Function

Both MRI and stereological means demonstrated pronounced intrarenal changes in the UO kidney compared to the control group [29]. For example, calculation of $V_{c}$ revealed a volume reduction of $28.9 \%$ in the obstructed kidney compared to $\mathrm{V}_{\mathrm{c}}$ of healthy kidneys. Stereology demonstrated that $\mathrm{N}_{\text {glom }}$ of UO kidneys was $18.2 \%$ reduced compared to the control group, suggesting that UO is followed by a substantial loss in nephrons. Interestingly, this $18.2 \%$ reduction in $\mathrm{N}_{\text {glom }}$ was accompanied by a $36.8 \%$ reduction in $\mathrm{V}_{\text {glom }}$ in the $\mathrm{UO}$ kidneys, suggesting loss of extracellular matrix. Next, we investigated the association between renal structural parameters and renal function. The structural parameters were renal $V_{c}$, total renal volume, $\mathrm{N}_{\text {glom }}$, and total $\mathrm{V}_{\text {glom }}$, and renal function was expressed by the single-kidney GFR (skGFR) obtained by ${ }^{51} \mathrm{Cr}$-EDTA clearance. Investigations were performed using the described UO pig model, and healthy pigs were considered as the control group. We found that skGFR overall correlated linearly with both $\mathrm{N}_{\text {glom }}$, $\mathrm{V}_{\text {glom }}$, $\mathrm{V}_{\mathrm{c}}$ and total kidney volume, with a coefficient of determination (based on linear fitting) in the range of $0.62-0.78$ [28]. Figure 3 shows the derived linear prediction with 95\% CI, and we believe that the observed correlations between structural parameters and renal function suggest that these parameters may potentially be useful as surrogate markers of renal function.

\section{Discussion and Conclusion}

Indeed, ultrasonography plays a critical role in many aspects of nephrological practice. The simplicity of this technique coupled with portability, low costs, and safety makes ultrasonography the modality of choice for kidney and vascular imaging. Nevertheless, disadvantages of ultrasonography include its operator dependence, limited imaging capability in patients with a large body habitus, 

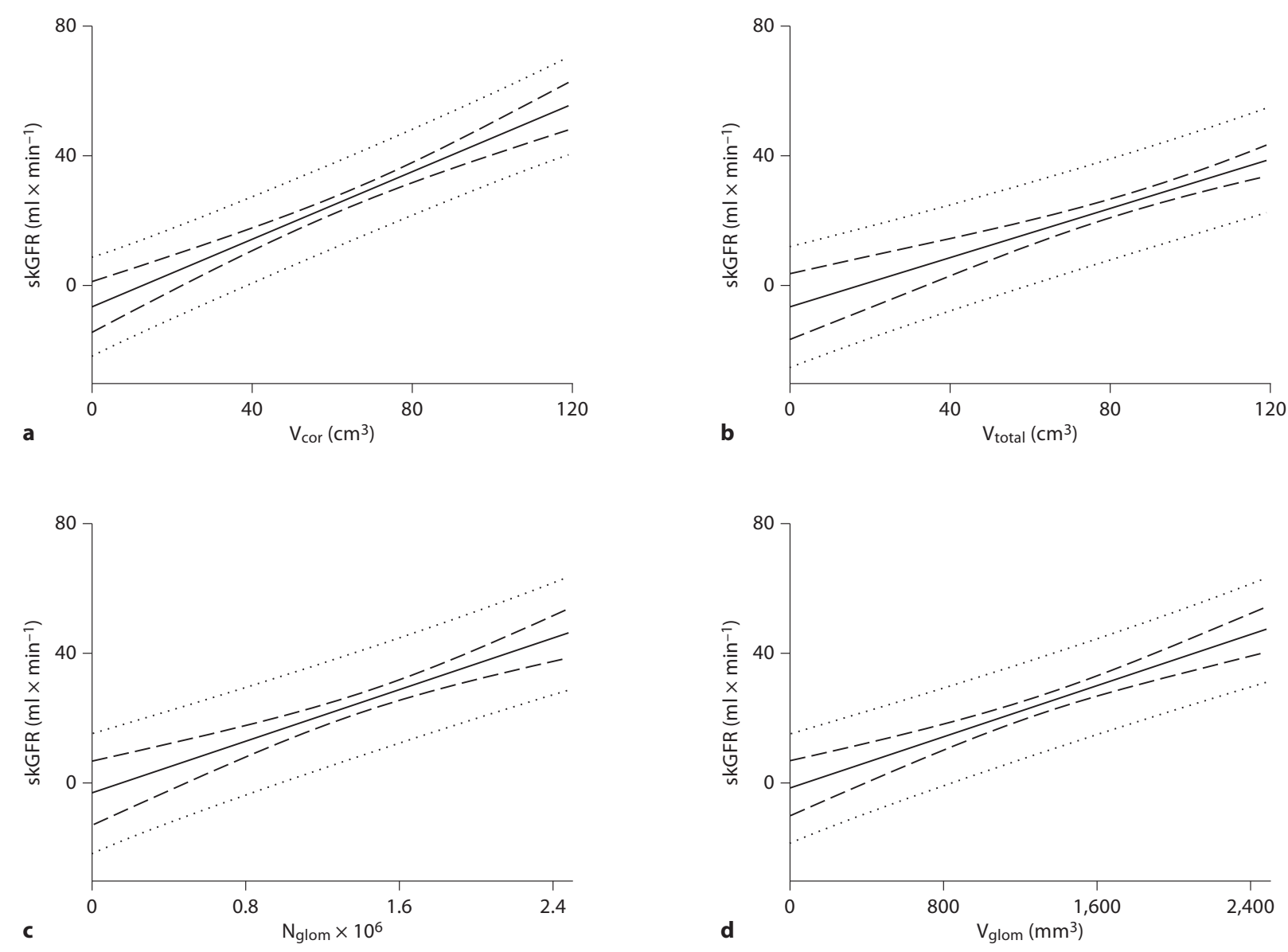

Fig. 3. Prediction of skGFR from the parameters $V_{\text {cor }}(\mathbf{a})$, total renal volume $\left(V_{\text {total }}\right)(\mathbf{b}), \mathrm{N}_{\text {glom }}(\mathbf{c})$, and total $V_{\text {glom }}$ (d). The full-drawn line indicates the best prediction, the long dashed lines indicate the 95\% CI limits, and the dotted lines indicate the $95 \%$ prediction interval limits.

decreased intrarenal sensitivity, and complete inability to assess renal function. We believe that calculated volumes of either the whole kidney or intrarenal segments represent the sum of general kidney growth and loss of nephron mass caused by associated renal disease. In current clinical practice, volume measurements are not usually used in routine examinations of renally impaired patients. Nonetheless, we refer to several renopathological conditions where volumetry would be clinically important because renal size gives insight into renal functional reserve or the extent of renal injury, including glomerulonephritis $[8,9]$, allograft nephropathy [10], parenchymal renal diseases [11], renal artery stenosis [12-14], and UO [19]. High-resolution MRI is capable of delineating particular intrarenal structures without radiation expo- sure and provides excellent tissue contrast. In addition, since multi-slice dynamic contrast-enhanced MRI with sufficient temporal resolution is now feasible by many clinical MRI devices, assessments of renal blood flow and renal function (clearance) can be evaluated in subjects with regional renal dysfunction [34]. A combination of intrarenal volumetric measures (such as $\mathrm{V}_{\text {glom }}$ ) with functional measures (filtration capacity per unit mass) allows a novel imaging-based method for important clinical data, such as skGFR [34].

We therefore suggest a complementary MRI volumetric approach without the need of contrast enhancement, combined with stereological analyses of biopsy samples. We have demonstrated that intrarenal measurements of $\mathrm{N}_{\text {glom }}, \mathrm{V}_{\text {glom }}$ and $\mathrm{V}_{\mathrm{c}}$ help to detect important structural 
and functional changes in a renally impaired animal model (UO pig model). In conclusion, we advocate a paradigm shift in current nephrological diagnostics where underlying structural and renophysiological changes in kidney disease are diagnostically evaluated on a quantitative intrarenal level.

\section{Disclosure Statement}

The authors declare that they have no conflicts of interest.

\section{References}

1 Hederström E, Forsberg L: Kidney size in children assessed by ultrasonography and urography. Acta Radiol Diagn 1985;26:85-91.

-2 Bauer MB, Miller SF, Ferrer FA, McKenna $\mathrm{PH}$ : Accuracy of renal ultrasound measurements for predicting actual kidney size. J Urol 1997;157:2278-2281.

- 3 Emamian SA, Nielsen MB, Pedersen JF, Ytte L: Kidney dimensions at sonography: correlation with age, sex, and habitus in 665 adult volunteers. Am J Roentgenol 1993;160:83-86.

4 Glodny B, Unterholzner V, Taferner B, Hofmann KJ, Rehder P, Strasak A, Petersen J: Normal kidney size and its influencing factors - a 64-slice MDCT study of 1,040 asymptomatic patients. BMC Urol 2009;9:19.

5 Nochy D, Lefaucheur C, Bariety J: Biopsie rénale: les différentes techniques. Nephrol Ther 2009;5:314-330.

6 6 Bonsib SM: Differential diagnosis in nephropathology: an immunofluorescence-driven approach. Adv Anat Pathol 2002;9:101-114.

7 Yong JL, Warren BA: A practical approach to the diagnosis of renal disease by biopsy. $\mathrm{Pa}$ thology 1994;26:370-396.

$\checkmark 8$ Danovitch GM, Nissenson AR: The role of renal biopsy in determining therapy and prognosis in renal disease. Am J Nephrol 1982;2:179-184.

-9 Wirta O, Mustonen J, Helin H, Pasternack A: Incidence of biopsy-proven glomerulonephritis. Nephrol Dial Transplant 2008;23:193-200.

10 Birnbaum LM, Lipman M, Paraskevas S, Chaudhury P, Tchervenkov J, Baran D, Herrera-Gayol A, Cantarovich M: Management of chronic allograft nephropathy: a systematic review. Clin J Am Soc Nephrol 2009;4: 860-865.

-11 Freedman BI, Iskander SS, Buckalew VM Jr, Burkart JM, Appel RG: Renal biopsy findings in presumed hypertensive nephrosclerosis. Am J Nephrol 1994;14:90-94.

\12 Gloviczki ML, Glockner JF, Lerman LO, McKusick MA, Misra S, Grande JP, Textor SC: Preserved oxygenation despite reduced blood flow in poststenotic kidneys in human atherosclerotic renal artery stenosis. Hypertension 2010;55:961-966.

13 Michel JB, Dussaule JC, Choudat L, Auzan C, Nochy D, Corvol P, Menard J: Effects of antihypertensive treatment in one-clip, two kidney hypertension in rats. Kidney Int 1986;29:1011-1020.
14 Tullis MJ, Zierler RE, Caps MT, Bergelin RO, Cantwell-Gab K, Strandness DE Jr: Clinical evidence of contralateral renal parenchymal injury in patients with unilateral atherosclerotic renal artery stenosis. Ann Vasc Surg 1998;12:122-127.

-15 Binkert CA, Hoffman U, Leung DA, Matter HG, Schmidt M, Debatin JF: Characterization of renal artery stenoses based on magnetic resonance renal flow and volume measurements. Kidney Int 1999;56:1846-1854.

16 Schrier RW: Diseases of the Kidney and Urinary Tract, ed 8 . Philadelphia, Lippincott Williams \& Wilkins, 2006.

17 Grantham JJ, Chapman AB, Torres VE: Volume progression in autosomal dominant polycystic kidney disease: the major factor determining clinical outcomes. Clin J Am Soc Nephrol 2006;1:148-157.

18 Kim HC, Yang DM, Jin W, Lee SH: Relation between total renal volume and renal function: usefulness of $3 \mathrm{D}$ sonographic measurements with a matrix array transducer. AJR Am J Roentgenol 2010;194:W186-W192.

19 Yoo KH, Thornhill BA, Forbes MS, Chevalier RL: Compensatory renal growth due to neonatal ureteral obstruction: implications for clinical studies. Pediatr Nephrol 2006;21: 368-375.

20 Thadhani R, Pascual M, Nickeleit V, TolkoffRubin N, Colvin R: Preliminary description of focal segmental glomerulosclerosis in patients with renovascular disease. Lancet 1996;247:231-233.

21 Rennke HG, Klein PS: Pathogenesis and significance of nonprimary focal and segmental glomerulosclerosis. Am J Kidney Dis 1989;13:443-456.

22 Bendtsen TF, Nyengaard JR: Unbiased estimation of particle number using sections - a historical perspective with special reference to the stereology of glomeruli. J Microsc 1989;153:93-102.

23 Nyengaard JR: Stereologic methods and their application in kidney research. Am Soc Nephrol 1999;10:1100-1123.

-24 Østerby R, Parving HH, Hommel E, Jørgensen HE, Løkkegaard H: Glomerular structure and function in diabetic nephropathy: early to advanced stages. Diabetes 1990;39:1057-1063.
25 Fulladosa X, Moreso F, Narváez JA, Grinyó JM, Serón D: Estimation of total glomerular number in stable renal transplants. J Am Soc Nephrol 2003;14:2662-2668.

26 Nyengaard JR, Gundersen HJ: Direct and efficient stereological estimation of total cell quantities using electron microscopy. J Microsc 2006;222:182-187.

27 Lødrup AB, Karstoft K, Dissing TH, Pedersen M, Nyengaard JR: Kidney biopsies can be used for estimations of glomerular number and volume: a pig study. Virchows Arch 2008;452:393-403.

28 Lødrup AB, Karstoft K, Dissing TH, Nyengaard JR, Pedersen M: The association between renal function and structural parameters: a pig study. BMC Nephrol 2008;9:18.

29 Karstoft K, Lødrup AB, Dissing TH, Sørensen TS, Nyengaard JR, Pedersen M: Different strategies for MRI measurements of renal cortical volume. J Magn Reson Imaging 2007;26:1564-1571.

30 Kobori H, Nangaku M, Navar LG, Nishiyama A: The intrarenal renin-angiotensin system: from physiology to the pathobiology of hypertension and kidney disease. Pharmacol Rev 2007:59:251-287.

31 Pedersen M, Dissing TH, Mørkenborg J, Stødkilde-Jørgensen H, Hansen LH, Pedersen LB, Grenier N, Frøkiaer J: Validation of quantitative BOLD MRI measurements in kidney: application to unilateral ureteral obstruction. Kidney Int 2005;67:2305-2312.

- 32 Eskild-Jensen A, Frøkiaer J, Djurhuus JC, Jørgensen TM, Nyengaard JR: Reduced number of glomeruli in kidneys with neonatally induced partial ureteropelvic obstruction in pigs. J Urol 2002;167:1435-1439.

-33 Bakker J, Olree M, Kaatee R, de Lange EE, Moons KG, Beutler JJ, Beek FJ: Renal volume measurements: accuracy and repeatability of US compared with that of MR imaging. Radiology 1999;211:623-628.

34 Mendichovszky I, Pedersen M, Frøkiaer J, Dissing T, Grenier N, Anderson P, McHugh $\mathrm{K}$, Yang Q, Gordon I: How accurate is dynamic contrast-enhanced MRI in the assessment of renal glomerular filtration rate? A critical appraisal. J Magn Reson Imaging 2008;27:925-931. 